\title{
Análise dos fatores relacionados à suspensão de operações em um serviço de cirurgia geral de média complexidade
}

\section{Analyses of the related factors for surgery suspension at a general surgery service of medium complexity}

Fábio Machado Landim(in memoriam) ${ }^{1}$; Francisco Diego Silva de Paiva ${ }^{2}$; Maria luciana Teles Fiuza33; Emanoelle Pinheiro de Oliveira ${ }^{4}$; Jonathan Guimarães Pereira ${ }^{5}$; Iana de Almeida Siqueira²

\section{R E S U M O}

\begin{abstract}
Objetivo: Identificar a taxa de suspensão de operações eletivas e investigar a ocorrência e as causas do seu cancelamento. Métodos: Trata-se de um estudo de natureza exploratória, descritiva, transversal e com abordagem quantitativa e qualitativa realizado no Departamento de Cirurgia Geral de um hospital de média complexidade. Foram analisadas todas as operações marcadas eletivamente no período compreendido entre janeiro de 2007 a julho de 2008. Resultados: No período do estudo, foram agendadas 7938 cirurgias, sendo 1806 urgências (22,75\%) e 6132 cirurgias eletivas (77,25\%). Do total de cirurgias eletivas, foram canceladas 1018, correspondendo a uma taxa de suspensão de $16 \%$. Os principais fatores dos cancelamentos das cirurgias estavam relacionados ao paciente (48,23\%), tais como condição clínica desfavorável (50,3\%) e não comparecimento (39,9\%); e ao hospital, tais como a alocação de recursos humanos $(23,77 \%)$, a organização da unidade $(22,88 \%)$ e a alocação de materiais e equipamentos $(5,12 \%)$. Conclusão: Verificou-se que $41 \%$ dos procedimentos suspensos não deveriam ter sido marcados. Medidas gerenciais corretivas são necessárias a fim de diminuir a taxa de suspensão.
\end{abstract}

Descritores: Procedimentos cirúrgicos operatórios. Cirurgia/organização \& administração. Centros de cirurgia. Controle de qualidade.

\section{INTRODUÇÃO}

$\mathrm{N}$ as últimas décadas, a suspensão de operações tem recebido atenção por parte de pesquisadores da área de saúde. A análise da taxa de suspensão das operações eletivas visa a melhoria da qualidade da assistência e da eficiência do serviço oferecido à população, assim como a racionalização dos recursos financeiros e humanos. Deve ter caráter periódico e ser divulgada para todos os envolvidos $^{1}$.

O Ministério da Saúde do Brasil $^{2}$ define a taxa de suspensão de cirurgia pelo número de cirurgias suspensas dividido pelo total de cirurgias programadas em determinado período e multiplicado por 100.

A taxa de suspensão de operações é um dos critérios de avaliação da eficiência de um serviço de cirurgia. Os baixos índices mostram o grau de desempenho do serviço avaliado e do centro cirúrgico da unidade hospitalar. Para a construção desse indicador devem ser considerados todos os motivos de suspensão de cirurgias ${ }^{1}$, como os relacionados ao paciente (condição clínica desfavorável, não comparecimento, falta de jejum e outras); e ao hospital, tais como organização da unidade (como erro na programação cirúrgica e prioridade para urgências) e alocação de recursos humanos e materiais.

Também se tem estudado a repercussão do cancelamento de cirurgia para o paciente e sua família. O procedimento cirúrgico causa alterações na rotina de vida de uma pessoa, com conseqüente afastamento das atividades desenvolvidas junto ao trabalho, ao lar e necessidade de mobilização de recursos financeiros, logo, o seu cancelamento gera frustração e perda econômica para o indivíduo e a sociedade 1 .

Do ponto de vista administrativo, a suspensão de uma cirurgia interfere na própria equipe de saúde e no consumo de tempo e de recursos materiais, indo, assim, de encontro à preocupação dos administradores da área da saúde, particularmente dos serviços cirúrgicos, em otimizar as atividades, reduzir gastos, evitar desperdícios e desenvolver o trabalho com a máxima qualidade ${ }^{3}$.

Este estudo tem como objetivo identificar a taxa de suspensão de operações eletivas e investigar as causas do seu cancelamento em serviço de cirurgia geral em hospital de média complexidade.

Trabalho realizado no Hospital Geral Dr. Waldemar Alcântara - Fortaleza, CE-BR.

1. Cirurgião Oncológico do Hospital Geral Dr. Waldemar Alcântara (HGWA)- FORTALEZA - CE-BR; 2. Alunos de Graduação em Medicina pela Universidade Estadual do Ceará (UECE)- Fortaleza - CE-BR; 3. Enfermeira Coordenadora do Centro Cirúrgico do Hospital Geral Dr. Waldemar Alcântara - Fortaleza, CE-BR; 4. Enfermeira Especialista em Gestão em Bloco Cirúrgico do Hospital Geral Dr. Waldemar Alcântara - Fortaleza, CE-BR; 5. Médico Residente em Cirurgia Geral do Hospital Geral Dr. Waldemar Alcântara - Fortaleza, CE-BR. 


\section{MÉTODOS}

Trata-se de um estudo de natureza exploratória, descritiva, transversal e com abordagem quantitativa e qualitativa realizado no Departamento de Cirurgia Geral do Hospital Geral Dr. Waldemar Alcântara (HGWA), localizado em Fortaleza, no estado do Ceará. Este é um hospital de média complexidade dentro da regionalização e do Sistema Único de Saúde, sendo gerido por uma organização social. O centro cirúrgico da unidade possui três salas cirúrgicas e realiza, em média, 380 a 400 procedimentos cirúrgicos/mês. Foram analisadas todas as cirurgias que integraram o mapa cirúrgico do período de 01 de janeiro de 2007 a 31 de julho de 2008. Foi definida como suspensão de operação programada, toda aquela que por qualquer razão não aconteceu. Eletiva ou de emergência.

Para verificar se houve cancelamento de cirurgias foi utilizado o banco de dados do prontuário eletrônico do departamento de Cirurgia Geral. O banco de dados é atualizado diariamente através do aviso de cirurgia, mapa cirúrgico, gastos de sala e formulários de suspensão. As causas de cancelamento das cirurgias foram classificadas pelos autores em relacionadas ao paciente (condição clínica desfavorável, não comparecimento, falta de jejum e outras); e ao hospital, tais como organização da unidade (como erro na programação cirúrgica e prioridade para urgências) e alocação de recursos humanos e materiais.

Para a análise estatística, os dados foram tabulados no programa Excel - Microsoft Office® 2003.

\section{RESULTADOS}

Das 7938 cirurgias agendadas no período de janeiro de 2007 a julho de 2008, foram 1806 urgências $(22,75 \%)$ e 6132 de caráter eletivo $(77,25 \%)$.

As operações foram classificadas segundo sua duração em: curta duração (menos de 30 minutos), média (de 30 a 90 minutos) e longa (mais de 90 minutos). A maioria delas foi de média duração $(46,2 \%)$, sendo seguida pelas de pequena $(44,5 \%)$ e longa duração $(9,3 \%)$ (Figura 1).

Durante o período estudado, do total de 6132 cirurgias programadas, foram realizadas 5114 cirurgias e suspensas 1018; logo, a taxa de suspensão foi de $16 \%$.

Neste estudo, como apresentado na tabela 1, das 1018 operações suspensas, tiveram como principal justificativa $(48,23 \%)$ problemas relacionados ao paciente, tais como condição clínica desfavorável (50,3\%) e não comparecimento (39,9\%).

Destacaram-se também as justificativas de operações suspensas relacionadas à organização da unidade $(22,88 \%)$, tais como prioridade para urgência $(72,1 \%)$ e erro na programação cirúrgica (12,5\%).

Problemas relacionados à alocação de recursos humanos $(23,77 \%)$ também foram responsáveis por uma parcela significativa de cancelamentos. Dentre eles, destacam-se o tempo cirúrgico excedido $(64,5 \%)$, a falta de anestesiologista (7,85\%), a falta de cirurgião $(6,2 \%)$ e a mudança de conduta médica (14,05\%).

As causas de suspensão de operações eletivas relacionadas à alocação de materiais e equipamentos representaram apenas $5,12 \%$ do total dos motivos de cancelamentos.

\section{DISCUSSÃO}

Um dos critérios de avaliação da eficiência de um serviço de cirurgia é a baixa taxa de suspensão de operações. Os baixos índices mostram o grau de desempenho do serviço avaliado e do centro cirúrgico da unidade hospitalar ${ }^{1}$. Para o hospital, o cancelamento de uma cirurgia eletiva implica em grandes prejuízos devido a ocupação do leito, a reserva da sala operatória, o desperdício de material cuja esterilização é dispendiosa, o pessoal envolvido no preparo de material e da sala cirúrgica e a perda da oportunidade de inclusão de outro paciente na programação cirúrgica ${ }^{3,4}$.

Para a construção desse indicador devem ser considerados todos os motivos de suspensão de cirurgias, tais como: falta de roupa, atraso do médico, falta de preparo do paciente, falta de jejum, não realização de exames prévios, extravio de radiografias e não cumprimento de um planejamento administrativo adequado' ${ }^{1}$.

Devem ser levados em consideração os aspectos psicológicos dos pacientes envolvidos. Estes demonstram revolta, desconfiança na equipe, insegurança, intenso estresse e aumento do nível de tensão. A comunicação do cancelamento cirúrgico tem sido motivo de preocupação por parte da equipe de saúde e da administração do hospital. A ocorrência deste evento muitas vezes é percebida como um fato corriqueiro e inerente à estrutura funcional da instituição. Tem sido crescente a preocupação e o cuidado ao comunicar o cancelamento. As explicações por vezes são incompletas e nem sempre coincidentes entre médicos e equipe de enfermagem ${ }^{1,4,5}$.

O controle adequado das cirurgias eletivas proporcionará menor espoliação do paciente, diminuição no

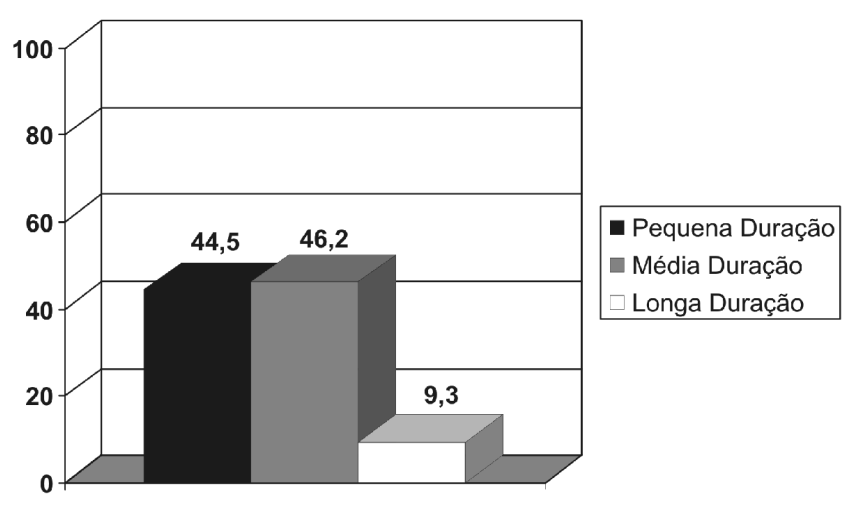

Figura 1 - Distribuição percentual das cirurgias agendadas e realizadas de acordo com a duração $(n=6920)$, Fortaleza-Ceará, 2007/08. 
Tabela 1 - Causas de suspensão de operações programadas (n=1018) - Fortaleza/Ceará - 2007/08.

\begin{tabular}{|c|c|c|c|c|}
\hline Causas & $\mathrm{n}$ & $\%$ & $\mathrm{~N}$ & $\%$ \\
\hline Relacionadas ao paciente & & & 491 & 48,23 \\
\hline Não comparecimento & 196 & 39,9 & & \\
\hline Condição Clínica Desfavorável & 247 & 50,3 & & \\
\hline Falta de Jejum & 22 & 4,5 & & \\
\hline Recusa à Realização de Cirurgia & 22 & 4,5 & & \\
\hline Intubação Difícil & 01 & 0,2 & & \\
\hline Paciente foi à Óbito & 03 & 0,6 & & \\
\hline Relacionadas à Organização da Unidade & & & 233 & 22,88 \\
\hline Falta de Vaga no CTI & 17 & 7,3 & & \\
\hline Prioridade para Urgência & 168 & 72,1 & & \\
\hline Falta de Exames & 17 & 7,3 & & \\
\hline Erro na programação cirúrgica & 29 & 12,5 & & \\
\hline Transferência para outro Hospital & 02 & 0,8 & & \\
\hline Relacionadas aos Recursos Humanos & & & 242 & 23,77 \\
\hline Falta de Hemoderivados & 06 & 2,5 & & \\
\hline Tempo Cirúrgico Excedido & 156 & 64,5 & & \\
\hline Falta de Anestesiologista & 19 & 7,85 & & \\
\hline Falta de Médico Auxiliar & 05 & 2 & & \\
\hline Falta de Cirurgião & 15 & 6,2 & & \\
\hline Preparo pré-operatório sem êxito & 04 & 1,65 & & \\
\hline Não Liberado pela Anestesista & 03 & 1,25 & & \\
\hline Mudança de conduta médica. & 34 & 14,05 & & \\
\hline Relacionadas aos Materiais e Equipamentos & & & 52 & 5,12 \\
\hline Falta de material & 16 & 30,7 & & \\
\hline Falta de Equipamentos & 36 & 69,3 & & \\
\hline Total & & & 1018 & 100,0 \\
\hline
\end{tabular}

tempo de internação e dos riscos de infecção hospitalar, redução dos custos do tratamento com benefícios diretos para os usuários que serão mais bem assistidos, e para a instituição que terá sua produtividade e retorno financeiros aumentados. Ao conhecer a taxa de suspensão de cirurgias e suas causas, a instituição pode evitar o seu cancelamento através de uma assistência de enfermagem planejada e articulada com as demais equipes profissionais e da elaboração de um planejamento de recursos de material e de pessoal ${ }^{4}$.

A taxa de suspensão de cirurgias neste estudo é de $16 \%$, coincidindo com as pesquisas realizadas por Paschoal e Gatto ${ }^{3}$ em um hospital universitário na cidade de São Paulo, onde a taxa foi de 19,91\%, e por Arieta el $a^{\kappa}$, que, em 200 pacientes oftalmológicos, foram encontradas 39 suspensões, apresentando taxa de 19,50\%. Outros estudos mostram taxas maiores de cancelamento de cirurgias. Gatto ${ }^{7}$ encontrou uma taxa de 27,33\% e Cavalcante et $a^{A}$ comenta que, após análise quantitativa, encontrou 33\% de suspensão de cirurgias em um hospital público e universitário da cidade de Fortaleza-Ceará. Segundo Cavalcante et al ${ }^{A}$ são necessárias maiores investigações nessa área, a fim de conhecer as causas e os motivos que determinam a suspensão das mesmas. Um hospital escola da província do Paquistão apresentou cerca de 25\% de suas cirurgias programadas suspensas ${ }^{8}$.
Outros estudos encontraram uma taxa de suspensão menor. Perroca et al' observou que, no período de três meses, foram programadas 4.870 cirurgias na unidade de Centro Cirúrgico de um hospital de ensino do interior de São Paulo, das quais 249 foram suspensas correspondendo a uma taxa de $5,1 \%$ de suspensões. Uma pesquisa realizada em um hospital terciário da Austrália observou que $13,2 \%$ das cirurgias eletivas foram canceladas no dia da realização do procedimento ${ }^{9}$. Lacqua e Evans ${ }^{10}$ ao analisarem prospectivamente 1063 casos de cirurgias programadas encontraram $184(17 \%)$ suspensões.

As taxas encontradas neste estudo contrariam o planejamento estratégico do HGWA, pois não deveriam exceder $10 \%$. Parte dessas suspensões foi devida à prioridade para urgências $(72,1 \%$ das causas relacionadas à organização da unidade) e ao tempo cirúrgico excedido $(64,5 \%$ das justificativas relacionadas aos recursos humanos); logo, são motivos não evitáveis inerentes ao serviço já que o percentual de urgências no período foi alto $(22,75 \%)$ e $9,3 \%$ dos procedimentos realizados foram de longa duração, fora do perfil destinado ao nível secundário.

Cada instituição deve estabelecer medidas específicas para reduzir, cada vez mais, a taxa de suspensão de cirurgia no seu serviço. Em nossa instituição há ambulatório de anestesia com o intuito de reduzir estes números, 
no entanto, em virtude da carência socioeconômica, os pacientes são internados sem apresentar as condições clínicas ideais para cirurgia.

Com relação às causas que contribuíram para a suspensão das cirurgias neste estudo, a maior parte relacionava-se ao paciente $(48,23 \%)$, seguida por aquelas relacionadas aos recursos humanos $(23,77 \%)$, à organização da unidade $(22,88 \%)$ e aos equipamentos e materiais $(5,12 \%)$. Em outra pesquisa a maioria das justificativas também se relacionava ao paciente $(57 \%)^{1}$. As causas relacionadas à organização da unidade $(22,1 \%)$ predominaram em relação à alocação de recursos humanos $(17,7 \%)$ e os motivos ligados aos equipamentos e materiais foram responsáveis por apenas 1,6\% das suspensões ${ }^{1}$. Outros estudos ${ }^{11,12}$ também mostram que as causas de suspensão de cirurgia estão fortemente atreladas a fatores intrínsecos ao paciente.

Dentre os fatores relacionados ao paciente, destacam-se a ausência de condições clínicas ideais (50,3\%) e o não comparecimento (39,9\%). Resultados semelhantes foram encontrados por Perroca et al', no entanto o não comparecimento do paciente à cirurgia agendada (56,3\%) predominou, indo ao encontro de em estudo realizado em um hospital universitário, no município de São Paulo ${ }^{3}$, onde a freqüência de operações canceladas pelo não comparecimento do paciente representou $54,3 \%$ do total das cirurgias canceladas.

No HGWA, as operações são agendadas durante a consulta ambulatorial, quando ocorre a avaliação clínica do paciente; logo, a marcação da cirurgia é, geralmente, realizada com muita antecedência, ficando o paciente susceptível às mudanças em suas condições clínicas o que pode inviabilizar o procedimento cirúrgico. Há, ainda, política de busca ativa, por telefone, da confirmação da presença dos pacientes no mapa, no dia anterior. Acreditamos que tanto a avaliação e a orientação pré-operatória mais efetiva ao paciente cirúrgico poderiam reduzir os valores encontrados.
Dentre as justificativas ligadas aos recursos humanos $(23,77 \%$ do total), destacaram-se o tempo cirúrgico excedido (64,5\%) e a mudança de conduta médica (14,05\%). Em um estudo realizado por Perroca et al' , a mudança de conduta médica $(43,2 \%)$ predominou em relação ao tempo cirúrgico excedido (25\%). Foram realizados no Centro Cirúrgico do HWGA 9,3\% de cirurgias de grande duração, contribuindo, assim, para um aumento no tempo cirúrgico. A falta de anestesiologista (7,85\%) também foi responsável pela suspensão de cirurgias. O preparo pré-operatório inapropriado foi responsável por apenas 1,65\% dos cancelamentos ligados aos recursos humanos, no entanto, esse fator pode ser evitado por uma melhor comunicação entre o paciente, os médicos e as enfermeiras.

Problemas relacionados à organização da unidade $(22,88 \%)$ também foram responsáveis por uma parcela significativas dos motivos de suspensão, destacando-se, dentre estes fatores, a prioridade para urgências (72,1\%). Esta é uma causa de suspensão inevitável tendo sido um importante fator devido a grande quantidade de cirurgias de urgências realizadas no período $(22,75 \%)$, apesar de não haver emergência aberta. As operações de urgência diminuem o número de leitos disponíveis e taxa de cirurgia em paciente eletivos, reduzindo, assim, a eficácia do serviço de cirurgia ${ }^{13}$.

Os resultados da presente pesquisa demonstraram que cerca de $41 \%$ dos procedimentos suspensos não deveriam ter sido marcados. Este número é ainda bastante alto dentro de um Serviço de Cirurgia Geral. Medidas de confirmação da presença dos pacientes que integram o mapa cirúrgico, ambulatório pré-anestésico, protocolos clínicos e informatização dos dados fizeram parte do programa de redução do número de suspensões, no entanto revisão dos processos e ferramentas de gestão como PDCA (composto pelos seguintes passos: Plan, Do, Check, Act Planejamento, Execução, Verificação e Ação) estão em realização para minimizar este transtorno e humanizar as relações entre os pacientes e seus médicos.

\title{
A B S S T R A C
}

\begin{abstract}
Objective: To identify the suspension rate on elective surgeries and to investigate their occurrences, and their cancelling causes. Methods: This is an exploratory, descriptive and cross-sectional study with a quantitative and qualitative approach which was carried out at the Department of General Surgery in a medium complexity Hospital. All surgeries performed from January 2007 to July 2008 were analyzed. Results: Seven thousand nine hundred and thirty eight surgeries were performed during the period of the research from which there were 1806 urgencies (22.75\%) and 6132 (77.25\%) were scheduled. There were 1018 canceled surgeries in the programmed group, corresponding to a cancellation rate of $16 \%$. Most of the reasons concerning cancellation were related to the patients (48.23\%), such as unfavorable clinical conditions (50.3\%) and absences (39.9\%); the reasons related to the hospital were because of human resources allocation $(23.77 \%)$, the organization of the unit $(22.88 \%)$ and the allocation of material and equipment (5.12\%). Conclusion: It was found that $41 \%$ of the canceled procedures should indeed not be performed. Some corrective management measures are necessary in order to diminish the cancellation rate.
\end{abstract}

Key words: Surgical procedures, operative. Surgery/organization \& administration. Surgicenters. Quality control.

\section{REFERÊNCIAS}

1. Perroca MG, Jericó MC, Facundin SD. Monitorando o cancelamento de procedimentos cirúrgicos: indicador de desempenho organizacional. Rev Esc Enferm USP. 2007; 41(1):113-9.
2. Ministério da Saúde. Secretaria Nacional de Organização e Desenvolvimento de Serviços de Saúde. Normas e padrões de construções e instalações do serviço de saúde. $2^{a}$ ed. Brasília; 1978.

3. Paschoal MLH, Gatto MAF. Taxa de suspensão de cirurgia em um hospital universitário e os motivos de absenteísmo do paciente à 
cirurgia programada. Rev Latino-am Enfermagem. 2006; 14(1):4853.

4. Cavalcante JB, Pagliuca LMF, Almeida PC. Cancelamento de cirurgias programadas em um hospital-escola: um estudo exploratório. Rev Latino-am Enfermagem. 2000; 8(4):59-65.

5. Antonio PS, Munari DB, Costa HK. Fatores geradores de sentimentos do paciente internado frente ao cancelamento de cirurgias. Revista Eletrônica de Enfermagem (online). 2002; 4(1):33-9. Disponível em: http://www.fen.ufg.br/

6. Lira RPC, Nascimento MA, Temporini ER, Kara-José N, Arieta CEL. Suspensão de cirurgia de catarata e suas causas. Rev Saúde Pública. 2001; 35(5): 487-9.

7. Gatto MAF. Análise da utilização de salas de operações [dissertação]. São Paulo -SP: Escola de Enfermagem/USP; 1996.

8. Zafar A, Mufti TS, Griffin S, Ahmed A, Ansari JA. Cancelled elective general surgical operations in Ayub teaching hospital. J Ayub Med Coll Abbottabad. 2007; 19(3):64-6.

9. Schofield WN, Rubin GL, Piza M, Lai YY, Sindhusake D, Fearnside MR, Klineberg PL. Cancellation of operations on the day of intended surgery at a major Australian referral hospital. Med J Aust. 2005; 182(12):612-5.

10. Lacqua MJ, Evans JT. Cancelled elective surgery: an evaluation. Am Surg. 1994; 60(11):809-11.
11. Lira RPC, Nascimento MA, Temporini ER, Kara-José N, Arieta CEL. Suspensão de cirurgias de catarata e suas causas. Saúde Pública 2001;35(5):487-9.

12. Arieta CEL, Taiar A, Kara-José N. Utilização e causas de suspensão de intervenções cirúrgicas oculares em Centro Cirúrgico ambulatorial universitário. Rev Assoc Méd Bras. 1995; 41(3):233-5.

13. Wyllie JH, Kidson IG, Wyllie DH. Pursuing efficiency in surgical practice. BMJ. 1988; 297(6660):1368-71.

Recebido em 03/11/2008

Aceito para publicação em 10/01/2009

Conflito de interesse: nenhum

Fonte de financiamento: nenhuma

\section{Como citar este artigo:}

Landim FM, Paiva FDS, Fiuza MLT, Oliveira EP, Pereira JG, Siqueira IA. Análise dos fatores relacionados à suspensão cirúrgica em um serviço de cirurgia geral de média complexidade. Rev Col Bras Cir. [periódico na Internet] 2009; 36(4). Disponível em URL: http://www.scielo.br/rcbc

Endereço para correspondência:

Francisco Diego Silva de Paiva

E-mail: diego_spaiva@hotmail.com 